\title{
DOUBLY SLICE KNOTS AND THE CASSON-GORDON INVARIANTS
}

BY

DANIEL RUBERMAN

\begin{abstract}
We find knots in all dimensions which are algebraically but not geometrically doubly slice. Our new obstructions involve the Casson-Gordon invariants of the finite cyclic covers in odd dimensions and of the infinite cyclic cover in even dimensions. These same invariants provide new criteria for amphicheirality and invertibility of even-dimensional knots.
\end{abstract}

0. Introduction. This paper investigates the question of double null-concordance of knots. Fox [7] originally raised the question as to when a knot is the slice of an unknot of the next higher dimension. If it is, it is called doubly null-concordant, or more concisely, doubly slice. Sumners $[24,25]$ gave an obstruction in terms of the Seifert form of an odd-dimensional knot, and showed (with a final step supplied by Kearton [14]) that his condition was, in fact, the only one for simple knots in high dimensions. He raised the question of what happens in even dimensions, in the nonsimple case, and in the classical dimension.

Stoltzfus [22, 23] treated the even-dimensional case and gave an obstruction based on the linking form defined by Levine [17] and Farber [6]. He remarked that for simple knots (with some restrictions) in high dimensions, his condition is sufficient to make a knot doubly slice. An obvious problem is to determine what happens for nonsimple knots and for low dimensions (e.g. knots in the four-sphere).

The principle result of this paper is that there are additional criteria for double null-concordance, and examples in all dimensions to show these criteria nonvacuous. In particular, we have

THEOREM. There are slice knots in $S^{2 k+1}(k \geqslant 1)$, satisfying Sumners' condition, which are not doubly slice. There are knots in $S^{2 k}(k \geqslant 2)$, satisfying Stoltzfus' condition, which are not doubly slice.

The above theorem for knots in $S^{3}$ is due to Pat Gilmer and Chuck Livingston [9]. Its proof will occupy $\S \S 1-4$.

The final section contains two further applications of Casson-Gordon invariants to high-dimensional knot theory. The first is to obtain new criteria for an even-dimensional knot to be ribbon. The second is to find new examples of noninvertible and nonamphicheiral knots, extending the work of Hillman [11]. 
1. Definitions and basic results. All knots and manifolds considered will be smooth, and will usually be considered as oriented also. Some notation will be used throughout.

$$
\left.\left(S^{n+2}, K\right) \text { (or just } K\right)=\text { a knotted } S^{n} \text { in } S^{n+2} .
$$

$X=S^{n+2}-K \times \dot{D}^{2}$ is the exterior of the knot, and $X_{\infty}$ will denote its infinite cyclic cover. Note that the choice of an orientation for $K$ and for $S^{n+2}$ implies a particular choice of meridian for $K$, and hence specifies a generator $t$ of the covering translations on $X_{x}$. In this way, $H_{*}\left(X_{x}\right)$ becomes a module over $\Lambda=\mathbf{Z}\left[t, t^{-1}\right]$. The linking form on the torsion of a manifold $M^{2 q+1}$ will be denoted by $\lambda: T_{k}(M) \times$ $T_{2 q-k}(M) \rightarrow \mathbf{Q} / \mathbf{Z}$. A $k n o t\left(S^{n+2}, K\right)$ is called simple if $\pi_{i}(X)=\pi_{i}\left(S^{1}\right)(i<n / 2)$.

Definition. A $\operatorname{knot}\left(S^{n+2}, K\right)$ is doubly slice if $K$ is the slice of an unknotted $S^{n+1}$ in $S^{n+3}$.

Let $V$ be the Seifert form associated to a Seifert surface $F$ for $\left(S^{2 n+1}, K\right) . V$ is hyperbolic if $H_{n} F \cong G_{1} \oplus G_{2}$ with $\operatorname{dim} G_{i}=\frac{1}{2} \operatorname{dim} H_{n} F$ and $V\left(G_{i} \times G_{i}\right)=0$. Sumners $[24,25]$ made the fundamental observation that if $K$ is doubly slice, then $K$ has a Seifert surface with a hyperbolic Seifert form. He also gave a partial converse, which Kearton strengthened.

THEOREM $1.1[25,14]$. If $K$ is a simple knot in $S^{2 k+1}(k>1)$ and $K$ has a Seifert surface with a hyperbolic Seifert form, then $K$ is doubly slice.

By analogy with ordinary knot concordance we have the following definition.

Definition. A knot in $S^{2 k+1}$ is algebraically doubly slice if it has a hyperbolic Seifert form.

In his study of knot modules [17], J. Levine defined, for a knot $\left(S^{2 q+2}, K\right)$, a pairing $L: T_{k}\left(X_{\infty}\right) \times T_{2 q-k}\left(X_{\infty}\right) \rightarrow \mathbf{Q} / \mathbf{Z}$. The definition of $L$ is complicated so we record its most important features: $L$ is a bilinear nonsingular form over $\Lambda$, and $L$ is $(-1)^{q+1}$-symmetric on $T_{q}\left(X_{\infty}\right)$. "Nonsingular" means that ad $L: T_{k}\left(X_{\infty}\right) \rightarrow$ $\operatorname{Hom}\left(T_{2 q-k}\left(X_{\infty}\right), \mathbf{Q} / \mathbf{Z}\right)$ is an isomorphism, while "over $\Lambda$ " means that $L(t x, t y)=$ $L(x, y)$. (An equivalent form was defined by M. S. Farber [6] in a somewhat more general context.)

Definition. $L$ is called hyperbolic if $T_{q}\left(X_{\infty}\right) \cong G_{1} \oplus G_{2}$ (as a $\Lambda$-module) with $L\left(G_{i} \times G_{i}\right)=0$.

Note that $G_{1} \cong G_{2}$ as abelian groups, but not as $\Lambda$-modules. In fact the $\Lambda$-structure on $G_{2}$ is the conjugate (interchange $t$ and $t^{-1}$ ) of the one on $G_{1}$.

Theorem 1.2 (Stoltzfus). If $\left(S^{2 q+2}, K\right)$ is doubly slice, then $L$ is hyperbolic.

Stoltzfus noted a converse for certain simple knots.

Definition. A $\operatorname{knot}\left(S^{2 q+2}, K\right)$ is an odd simple knot if $K$ is simple, and $H_{q}\left(X_{\infty}\right)$ has no 2-torsion.

Kearton [15] classified such knots when $q>2$. Stoltzfus [22] states (without proof) that using Kearton's classification one can show

THEOREM 1.3. If $\left(S^{2 q+2}, K\right)$ is an odd simple knot such that $L$ is hyperbolic and $q>2$, then $K$ is doubly slice. Again, by analogy, $K$ is defined to be algebraically doubly slice if $L$ is hyperbolic. 
The main results of this thesis use the Casson-Gordon invariants $[4,5]$ defined below.

For $d$ an integer, set $\omega=e^{2 \pi i / d}$, and suppose $\phi: A \rightarrow B \mathbf{Z}_{d}$ is a map. Then $\phi$ corresponds to a class $\phi \in H^{1}\left(A ; \mathbf{Z}_{d}\right)=\operatorname{Hom}\left(H_{1}(A) ; \mathbf{Z}_{d}\right)$ and induces a cyclic cover $\tilde{A} \rightarrow A$ with a specific choice $T: \tilde{A} \rightarrow \tilde{A}$ of a generator of the covering translations. Let $\bar{H}_{k}(A, \phi)=\omega$-eigenspace of $T_{*}$ acting on $H_{k}(\tilde{A} ; \mathbf{C}), \bar{\beta}_{k}(A, \phi)=$ $\operatorname{dim}_{\mathrm{C}} H_{k}(A, \phi)$ and $\bar{\chi}(A, \phi)=\Sigma(-1)^{k} \bar{\beta}_{k}(A, \phi)$. If $A$ is a compact $2 k$-manifold, then the intersection form on $A$ induces a Hermitian pairing on $H_{k}(\tilde{A} ; \mathbf{C})=H_{k}(\tilde{A}) \otimes \mathbf{C}$ : for $k$ even, $\langle x \otimes \alpha, y \otimes \beta\rangle=x \cdot y \otimes \alpha \bar{\beta}$, and for $k$ odd $\langle x \otimes \alpha, y \otimes \beta\rangle=x \cdot y \otimes$ $i \alpha \bar{\beta}$. Define $\bar{\sigma}(A, \phi)=$ the signature of $\langle$,$\rangle restricted to \bar{H}_{k}(A, \phi)$. If the character $\phi$ is clear from the context, we will simply write $\bar{H}_{k} A$ for $\bar{H}_{k}(A, \phi)$, etc.

Now suppose $M$ is a (closed) $(2 k-1)$-manifold and $\phi \in H^{1}\left(M ; \mathbf{Z}_{d}\right)$; by bordism theory $n \cdot(M, \phi)=\partial(W, \phi)$ for some $W$.

Definition. $\sigma(M, \phi)=(\bar{\sigma}(W, \phi)-\sigma(W)) / n$; it can be shown that this depends only on $M$ and $\phi$. For example, the bordism argument given in [8] applies as well in any dimension.

We will need the following propositions of Smith theory due to Gilmer [8]. Let $\phi$ : $H_{1}(A) \rightarrow \mathbf{Z}_{d}$ be a character where $d=p^{r}$ is a power of a prime and $A$ is a complex. $A$ is finite-dimensional, though not necessarily compact. However, for each $n$ with $0 \leqslant n \leqslant r$, assume that the induced $p^{n}$-fold cover of $A$ has finitely generated homology with rational coefficients, and that $H_{*}\left(A ; \mathbf{Z}_{p}\right)$ and $H_{*}\left(\tilde{A}, \mathbf{Z}_{p}\right)$ are finite. With these hypotheses we have

Proposition 1.4. $\bar{\chi}(A)=\chi(A)$.

Proposition 1.5. $\bar{\beta}_{j}(A) \leqslant \beta_{j}\left(A ; \mathbf{Z}_{p}\right)$ for all $j$.

Proposition 1.6. If $\tilde{A}$ is connected, then $\bar{\beta}_{1}(A) \leqslant \beta_{1}\left(A, \mathbf{Z}_{p}\right)-1$.

Propositions 1.4-1.6 correspond to $1.1,1.4$ and 1.5 of [8]. They are proved in [8] in the case that $A$ is compact, but Gilmer's proofs go through in the present generality once we note the following facts.

(1) The Smith homology groups $H^{\delta^{m}}(\tilde{A})$ used in [8] are finite. This follows by induction from the long exact sequence

$$
\rightarrow H_{k}^{\delta}(\tilde{A}) \rightarrow H_{k}^{\delta^{m}}(\tilde{A}) \rightarrow H_{k}^{\delta^{m-1}}(\tilde{A}) \rightarrow H_{k-1}^{\delta}(\tilde{A}) \rightarrow,
$$

and the observation that $H_{*}^{\delta}(\tilde{A})=H_{*}\left(A, \mathbf{Z}_{p}\right)$ which is finite.

(2) The arguments of Thomas and Wood [26] used in [8] apply in the present context, for compactness is never used in the relevant section (\$7) of [26].

(3) The proof of Proposition 1.1 in [8] uses the fact that the Euler characteristic multiplies under finite covers. This fact is proved by a spectral sequence argument in our situation (i.e. finitely generated rational homology) in Spanier [21].

2. The basic approach. The problem at hand is to find invariants beyond those of Stoltzfus and Sumners for nonsimple knots, i.e. to find algebraically but not geometrically doubly slice knots. The odd-dimensional case is simpler and motivates the approach to the even-dimensional case, so we discuss it first. 
Observation. If $\left(S^{n+2}, K\right)$ is doubly slice, then any cyclic covering of $S^{n+2}$ branched along $K$ imbeds in $S^{n+3}$. This is easy to see: the branched covering of $\left(S^{n+2}, K\right)$ extends to a branched covering of the unknotted $\left(S^{n+3}, S^{n+1}\right)$. But this latter is just $S^{n+3}$. So obstructions to codimension-one imbeddings give rise to obstructions to double null-concordance. This approach was used by Gilmer and Livingston [9], who showed

THEOREM 2.1. Let $M^{3}$ be a rational homology 3-sphere. If $M \subset S^{4}$, then $H_{1} M=G_{1}$ $+G_{2}$ with (1) $G_{1} \cong G_{2}$, (2) $\lambda_{\mid G_{i}}=0$, and (3) for all $\phi: H_{1} M \rightarrow \mathbf{Z}_{d}(d=$ a power of the prime $p$ ) with $\phi_{\mid G_{1}}=0$ or $\phi_{\mid G_{2}}=0,|\sigma(M, \phi)|+\bar{\beta}_{1}(M, \phi)<\beta_{1}\left(M ; \mathbf{Z}_{p}\right)$.

They used Theorem 2.1 to give examples of slice knots in $S^{3}$ which are algebraically doubly slice, but whose 2 -fold covers do not imbed in $S^{4}$.

In the same spirit, we have

THEOREM 2.2. Let $M^{2 k+1}$ be a rational homology sphere. If $M \subset S^{2 k+2}$, then $H_{*}(M)=G_{*}+G_{*}^{\prime}(* \neq 0,2 k+1)$ with (1) $G_{k} \cong G_{k}^{\prime}$, (2) $\lambda_{\mid G_{k}}=\lambda_{\mid G_{k}^{\prime}}=0$, and (3) $\forall \phi: \quad H_{1} M \rightarrow \mathbf{Z} / d\left(d=p^{r}\right)$ with $\phi_{\left.*\right|_{i *}}=0$ or $\phi_{\left.*\right|_{i *}}=0$, we have $|\sigma(M, \phi)| \leqslant$ $\beta_{k}\left(M ; \mathbf{Z}_{p}\right)$. (In condition $3, \phi_{*}$ is defined by considering $\phi$ as a map $\phi: M \rightarrow B \mathbf{Z}_{d}$ and taking the induced map on homology.)

Theorem 2.2 provides examples in every odd dimension of algebraically but not geometrically doubly slice knots. The condition that $\phi_{* k_{i *}}=0$ for $*>1$ can be of some use because it restricts the possible characters for which one must compute $\sigma(M, \phi)$. This principle is used in [20] to study the problem of imbedding connected sums of lens spaces and imbedding punctured lens spaces. In that paper, the fact that the linking form splits hyperbolically restricts the characters considerably.

The even-dimensional case starts from a similar observation. If $\left(S^{2 q+2}, K\right)$ is doubly slice, then $\left(X_{\infty}, \partial X_{\infty}\right)=\left(X_{\infty}, S^{2 q} \times \mathbf{R}\right) \subset\left(B^{2 q+2} \times \mathbf{R}, S^{2 q+1} \times \mathbf{R}\right)$. The reason is as above: the $\mathbf{Z}$-cover of $X$ extends to the $\mathbf{Z}$-cover of $S^{2 q+3}-S^{2 q+1} \times \dot{D}^{2}$, which is $B^{2 q+2} \times \mathbf{R}$. Consider now the special case where $K$ is fibered with fiber $M_{0}$; set $M=M_{0} \cup B^{2 q+1}$. Then $X_{\infty}=M_{0} \times \mathbf{R}$, so that if $K$ is doubly slice, then $\left(M_{0} \times \mathbf{R}, \partial M_{0} \times \mathbf{R}\right) \subset\left(B^{2 q+2} \times \mathbf{R}, S^{2 q+1} \times \mathbf{R}\right)$. Capping off, we then have $M \times \mathbf{R}$ $\subset S^{2 q+2} \times \mathbf{R}$, and it seems as though one should "cancel the $\mathbf{R}$-factor" to obtain $M \subset S^{2 q+2}$. Theorem 2.1 or 2.2 , applied to the fiber $M$, would then be an obstruction to $K$ being doubly slice.

Something like this turns out to be true, although we do not actually show that the capped-off fiber imbeds in $S^{2 q+2}$. Roughly speaking, for $\left(S^{2 q+2}, K\right)$ a knot with $H_{*}\left(X_{\infty}, \mathrm{Q}\right)=0$, if $K$ is doubly slice then one has the conclusion of Theorem 2.1 (if $q=2$ ) or Theorem 2.2 (if $q>2$ ) with $X_{\infty}$ playing the role of $M$. However, in (3), $\sigma(M, \phi)$ is replaced by $\sigma(M, \phi \circ i)$ where $\phi: H_{1}\left(X_{\infty}\right) \rightarrow \mathbf{Z}_{d}$ is a character and $i$ : $M_{0} \rightarrow X_{\infty}$ is a lift of $M_{0}$ to the cover $X_{\infty}$. This provides infinitely many new examples of non-doubly slice knots in every even dimension.

The same idea of using the Casson-Gordon invariants of a Seifert surface is applied in the final section. There we find new criteria for a knot to be ribbon, and new obstructions to amphicheirality and invertibility. For ribbon knots, all the invariants on $M$, coming from characters on $X_{\infty}$, must vanish. To detect invertibility 
and amphicheirality, we examine the effect of changing the orientation of the knot and of the ambient sphere on the Casson-Gordon invariants.

3. The odd-dimensional case. In this section, we investigate the imbedding of $(2 k+1)$-dimensional manifolds in $S^{2 k+2}$, and use these results to find new nondoubly slice knots in $S^{2 k+1}(k>1)$. The main tool is Theorem 2.2 which we now prove.

Proof (OF 2.2). If $M$ is imbedded in $S^{2 k+2}$, it separates $S^{2 k+2}$ into two components $W$ and $W^{\prime}$. For $* \neq 0,2 k+1$, let $G_{*}=\operatorname{ker} H_{*}(M) \rightarrow H_{*}(W)$ and $G_{*}^{\prime}=\operatorname{ker} H_{*}(M) \rightarrow H_{*}\left(W^{\prime}\right)$. It is easy to see that $\lambda_{\mid G_{*}}=0$, and, likewise, that $\lambda_{\mid G_{*}^{\prime}}=0$. It follows from the Mayer-Vietoris sequence that $H_{*}(M)=G_{*}+G_{*}^{\prime}$, and from duality that $G_{k} \cong G_{k}^{\prime}$. (See [9 or 10] for the argument.)

Suppose, say, that $\phi_{\left.*\right|_{i_{*}}}=0$. Then $\phi$ extends to a character $\phi: H_{1}(W) \rightarrow \mathbf{Z}_{d}$, so we can use $W$ to calculate $\sigma(M, \phi)$. By definition $\sigma(M, \phi)=\bar{\sigma}(W)-\sigma(W)$; since $H_{k+1}(W ; \mathbf{Q})=0, \sigma(W)=0$. There is the obvious inequality that $|\bar{\sigma}(W)| \leqslant \bar{\beta}_{k+1}(W)$. By Proposition $1.5, \bar{\beta}_{k+1}(W) \leqslant \operatorname{dim} H_{k+1}\left(W ; \mathbf{Z}_{p}\right)$. But the Mayer-Vietoris sequence with $\mathbf{Z}_{p}$-coefficients shows that

$$
H_{k+1}\left(W ; \mathbf{Z}_{p}\right)+H_{k+1}\left(W^{\prime} ; \mathbf{Z}_{p}\right)=H_{k+1}\left(M ; \mathbf{Z}_{p}\right),
$$

so

$$
|\boldsymbol{\sigma}(M, \chi)| \leqslant \operatorname{dim} H_{k+1}\left(M ; \mathbf{Z}_{p}\right) .
$$

Theorem 2.2 provides examples of non-doubly slice knots in $S^{2 k+1}$. A similar method was used in the classical dimension by Gilmer and Livingston so we confine ourselves to $k>1$. We will see shortly that the 2-twist spins of these knots are not doubly slice, completing the main goal of this paper. Here is the construction, which works in any dimension.

Let $\left(S^{n+2}, J\right)(n>1)$ be a knot with $\pi_{1}\left(S^{n+2}-J\right)=\mathbf{Z}$ and $*$ a base point on $J$. Imbed $J \times[-1,1]$ in $S^{n+2}$ by using a normal vector field; since $n>1$ this is unique. Let $q$ be an integer, and form the knot $K=K(J, q)$ by connecting $* \times\{-1\}$ to $* \times\{1\}$ by a 1-handle that links $J \times\{0\} q$ times. The 1-handle should be imbedded with no twisting, i.e. so that the framing on the resulting $S^{1}$ extends over a disc. This imbeds $F^{n+1} \cong S^{n} \times S^{1}-D^{n+1}$ in $S^{n+2}$; let $K=\partial F$.

Note the following properties of $K(J, q)$.

Proposition 3.1. (a) $K(J, 0)$ is the unknot. (b) $K$ is a slice knot. (c) If $J$ is slice then $K$ is doubly slice. (d) $K$ is algebraically doubly slice.

Proof. (a) If $q=0$, then $S^{1} \subset F$ bounds a disc $D^{2}$ in $S^{n+2}-F$ which we can surger to get $K=\partial D^{n+1}$.

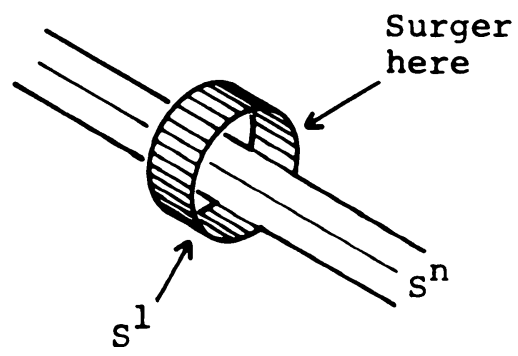


(b) Even if $q \neq 0$, we can ambiently surger the $S^{1}$ in $B^{n+3}$ to form a slice disc for $K$.

(c) If $J$ is slice, we can surger it in, say, $B_{+}^{n+3}$ and the $S^{1}$ in $B_{-}^{n+3}$ (here $S^{n+3}=B_{+}^{n+3} \cup B_{-}^{n+3}$ ). The traces of these two surgeries fit together to form a copy of $D^{n+2} \subset S^{n+3}$ whose intersection with $S^{n+2}$ is exactly $F$. Hence, $K=\partial F$ is doubly slice.

(d) For $n$ even this is easy: $J$ is always slice [16] so $K(J, q)$ is doubly slice, hence algebraically so. For $n$ odd it is just as easy. The Seifert form is empty (hence hyperbolic!) since $n>1$.

Since $K(J, q)$ is doubly slice for $n$ even, we confine ourselves to $n$ odd. It seems plausible to conjecture that the converse to (c) holds, i.e. that if $K(J, q)$ is doubly slice, then $J$ is slice. Using Theorem 2.2 we obtain a weak version of this converse-if $K$ is doubly slice then certain concordance invariants of $J$ must vanish.

Definition. Let $\left(S^{2 k+1}, J\right)$ be a knot or link and $V$ a Seifert matrix of $J$. Set $\omega=e^{2 \pi i / q}$. Define $\sigma_{p / q}(J)=\operatorname{sign}\left(\left(1-\omega^{p}\right) V+\left(1-\omega^{-p}\right) V^{\prime}\right)$.

THEOREM 3.2. Suppose $2 q+1=p^{r}$ ( $p$ a prime) and let $\left(S^{2 k+1}, J\right)$ be a knot $(k>1)$. If $K(J, q)$ is doubly slice, then

(1) If $k$ is even $\sigma_{s / 2 q+1}(J)=0(0<s<2 q+1)$.

(2) If $k$ is odd

$$
\sigma_{s / 2 q+1}(J)+2^{2 k-2} \frac{2 q+1-2 s}{2 q+1} \sigma_{1 / 2}(J)=0 \quad(0<s<2 q+1) .
$$

Proof. Let $M=$ the two-fold branched cover of $\left(S^{2 k+1}, K\right)$, and suppose $K$ is doubly slice. As noted in the introduction, $M$ must therefore imbed in $S^{2 k+2}$. So we can apply Theorem 2.2 to $M$. By definition, $K$ has its Seifert surface $F=S^{2 k-1} \times S^{1}$ $-D^{2 k}$; if $\hat{F}$ denotes $F$ pushed into $B^{2 k+2}$ except along $K$, we can write $M=\partial W$ where $W=2$-fold cover of $\left(B^{2 k+2}, \hat{F}\right)$.

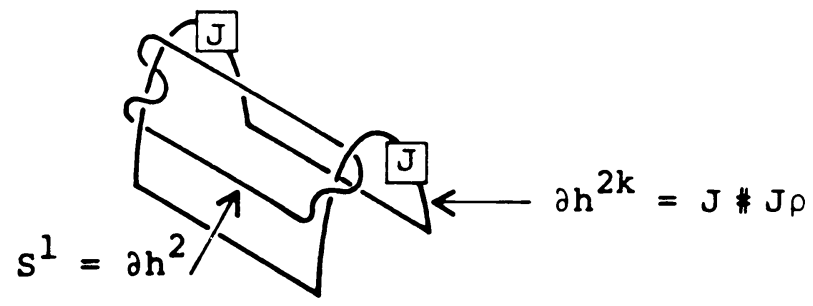

The well-known cut and paste description of $W[13]$ shows how to build $W$ as a union of two $(2 k+2)$-balls glued along copies of $F$. The analysis (given in [1] for $k=1$ ) of Akbulut and Kirby applies as well in any dimension and yields the following "framed link" picture of $W: W=B^{2 k+2} \cup h^{2} \cup h^{2 k}$. The handle $h^{2 k}$ is added along the knot $J \# J \rho$ ( $J \rho=J$ with reversed orientation) and $h^{2}$ is added along a circle $S^{1}$ which links $J$ \# $\rho$ exactly $2 q+1$ times. See the above figure. Hence $H_{1}(M) \cong \mathbf{Z}_{2 q+1}=\mathbf{Z}_{p^{r}}$ generated by a meridian $\mu$ of $J \# J \rho$. Apply Theorem 2.2 to $M$ - the only way to split up $H_{1}(M)$ into $G_{1}+G_{1}^{\prime}$ is for $G_{1}=\mathbf{Z}_{p^{r}}$ and $G_{1}^{\prime}=0$. So take the character $\phi: H_{1}(M) \rightarrow \mathbf{Z}_{p^{r}}$ defined by $\phi(\mu)=1$. Since $K$ is doubly slice, 
$M$ imbeds in $S^{2 k+2}$ and so, by Theorem $2.2,|\sigma(M, s \phi)| \leqslant \operatorname{dim} H_{k}\left(M ; \mathbf{Z}_{p}\right)=0$ for all $s$. It will be shown in Theorem 3.5 that

$$
\sigma(M, s \phi)=\left\{\begin{array}{l}
\sigma_{s / 2 q+1}(J \# J \rho) \quad(k \text { odd }), \\
\sigma_{s / 2 q+1}(J \# J \rho)+2^{2 k-2} \frac{2 q-2 s+1}{2 q+1} \sigma_{1 / 2}(J \# J \rho) \quad(k \text { even }) .
\end{array}\right.
$$

But $J$ and $J \rho$ have the same Seifert surface, except with reversed orientation, hence a Seifert form for $J \rho$ is just the transpose of that for $J$. Taking the transpose does not affect the signatures; by additivity

$$
\sigma_{s / 2 q+1}(J \# J \rho)=2 \sigma_{s / 2 q+1}(J) \text { and } \sigma_{1 / 2}(J \# J \rho)=2 \sigma_{1 / 2}(J),
$$

so the theorem follows.

COROLlary 3.3. In each $S^{2 k+1}(k>1)$ there are infinitely many slice knots, which are algebraically but not geometrically doubly slice.

Proof. The different signatures $\sigma_{s / 2 q+1}$ are independent so we can construct infinitely many (different) $K(J, q)$.

To complete the proof of Theorem 3.2 we need to see how to compute the Casson-Gordon invariants of manifolds such as $M$, which arose in its proof. The answer is provided by Theorem 3.5 below which shows how to compute $\sigma(M, \phi)$ when the covering $\tilde{M} \rightarrow M$ extends to a branched covering rather than an unbranched one. The technique is due to Casson and Gordon [5] who consider the case $k=1$; we will consider $k>1$.

LeMma 3.4. Suppose $\tilde{N}^{2 m} \rightarrow N^{2 m}$ is a branched $\mathbf{Z}_{d}$-cover of the closed manifold $N$ with generator $t$ of the covering translations corresponding to the character $\phi: H_{1}(N) \rightarrow$ $\mathbf{Z}_{d}$. If the branch set $F^{2 m-2}$ has trivial normal bundle, then $\forall 0<r<d$,

$$
\bar{\sigma}(N, r \phi)=\sigma(N)-2^{2 m-2} \frac{d-2 r}{d} \sigma(F) .
$$

Proof. Let $\operatorname{sign}(g, \tilde{N})$ denote the $g$-signature [2] of the $\mathbf{Z}_{d}$-action on $\tilde{N}$. The argument in [5, Lemma 2.1] is valid for any $m$ (including $m$ odd) and shows that

$$
\bar{\sigma}(N, r \phi)=\sigma(N)+\frac{1}{d} \sum_{s=1}^{d-1}\left(\omega^{-r s}-1\right) \operatorname{sign}\left(t^{s}, \tilde{N}\right) \quad\left(\omega=e^{2 \pi i / d}\right) .
$$

Since $\nu(\tilde{F}, \tilde{N})$ is trivial, the $G$-signature theorem says

$$
\operatorname{sign}\left(t^{s}, \tilde{N}\right)=2^{2 m-2} i \tan (\pi s / d) \sigma(F) .
$$

This proves the lemma when $m$ is even, where $\sigma(F)=0$. For $m$ odd, we have

$$
\bar{\sigma}(N, r \phi)=\sigma(N)-\frac{2^{2 m-2}}{d} i \sigma(F) \sum_{s=1}^{d-1}\left(\omega^{-r s}-1\right) \cot \frac{\pi s}{d} .
$$

Now

$$
\sum_{s=1}^{n-1}\left(\omega^{-r s}-1\right) \cot \frac{\pi s}{d}=\sum_{s=1}^{n-1} \cos \left(\frac{2 \pi r s}{d}-1\right) \cot \frac{\pi s}{d}-i \sum_{s=1}^{n-1} \sin \frac{2 \pi r s}{d} \cot \frac{\pi s}{d}
$$


It is easy to verify that the real part vanishes; the imaginary part is

$$
\sum_{s=1}^{n-1} \sin \frac{2 \pi r s}{d} \cot \frac{\pi s}{d}=-2 d\left(\frac{r}{d}-\left[\frac{r}{d}\right]-\frac{1}{2}\right)
$$

by a formula of Eisenstein (see [28, p. 103]). Since $r<d$, this is just $d-2 r$, so

$$
\bar{\sigma}(N, r \phi)=\sigma(N)-2^{2 m-2} \frac{d-2 r}{d} \sigma(F) .
$$

THEOREM 3.5. Let $L^{2 m-1}=\left\{L_{j}\right\}$ be a link in $S^{2 m+1}(m>1)$ and let $W=B^{2 m+2}$ $\cup\left\{h_{i}^{2}\right\} \cup\left\{h_{j}^{2 m}\right\}$, where the handle $h_{j}^{2 m}$ is attached along $L_{j}$ and $\left\{h_{i}^{2}\right\}$ is some collection of 2-handles. Let $M=\partial W$, and suppose $\phi: H_{1}(M) \rightarrow \mathbf{Z}_{d}$ is the character that gives 1 on the meridian of each $L_{i}$. Then $\forall 0<r<d$,

$$
\sigma(M, r \phi)=\left\{\begin{array}{l}
\sigma_{r / d}(L) \quad(m \text { odd }), \\
\sigma_{r / d}(L)+2^{2 m-2} \frac{d-2 r}{d} \sigma_{1 / 2}(L) \quad \text { (m even). }
\end{array}\right.
$$

Proof. Let $F_{0}$ be any Seifert surface for $L$, and let $F=F_{0} \cup_{L}$ \{cores of the $\left.h_{j}^{2 m}\right\}$. Then the cover of $m$ induced by $r \phi$ extends to a cover $\tilde{W} \rightarrow{ }^{\pi} W$ branched along $F$; note that $\nu(F)$ is trivial. Novikov additivity for $\bar{\sigma}($,$) and the previous lemma imply$ that $\sigma(M, r \phi)$ can be calculated using this branched cover: i.e.

$$
\sigma(M, r \phi)=\bar{\sigma}(W, r \phi)-\sigma(W)+2^{2 m-2} \frac{d-2 r}{d} \sigma(F) .
$$

Now $W$ splits up into $\tilde{B} \cup \tilde{H}$, where $\tilde{B}=\pi^{-1} B$ and $\tilde{H}=\pi^{-1}\left(\left\{h_{i}^{2}\right\} \cup\left\{h_{j}^{2 m}\right\}\right)$. It is easy to see that $\tilde{H}$ makes no contribution to $\bar{\sigma}(W)$. Hence $\bar{\sigma}(W, r \phi)=\bar{\sigma}(B, r \phi)=$ $\sigma_{r / d}(L)$ by the well-known interpretation of $\sigma_{r / d}(L)$ as an eigenspace signature. Since $m>1$, there is no middle-dimensional homology (i.e. $H_{m+1}(W)=0$ ) so that $\sigma(W)=0$. Finally $\sigma_{1 / 2}(L)=\sigma(F)$ if $m$ is even, and so the theorem follows.

4. Infinite cyclic covers. The purpose of this section is to use the Casson-Gordon invariants to study double null-concordance of even-dimensional knots. Recall the main geometric idea from the introduction. $X_{\infty}$, the Z-cover of $S^{2 k-2}-K \times D^{2}$, resembles, in some sense, a $(2 k+1)$-manifold cross $\mathbf{R}$. If $K$ is doubly slice, then it appears that this $(2 k+1)$-manifold imbeds properly in $B^{2 k+2}$. Hence, the obstructions to codimension-one imbeddings developed in $\$ 2$ might be obstructions to double null-concordance. In order to make this idea work we investigate infinite cyclic covers in order to see that they have Casson-Gordon invariants just like compact manifolds (at least in certain situations).

For this section, $\left(Y_{\infty}, \partial Y_{\infty}\right)$ will denote an infinite cyclic cover of a compact manifold $\left(Y^{n}, \partial Y^{n}\right)$, and $p$ will be a prime number.

TheOREM 4.1 (Milnor [18]). Let $F$ be a field, and suppose $H_{*}\left(Y_{\infty} ; F\right)$ and $H_{*}\left(\partial Y_{\infty} ; F\right)$ are finitely generated. Then $H_{n-1}\left(Y_{\infty}, \partial Y_{\infty} ; F\right) \cong H^{n-1}\left(Y_{\infty}, \partial Y_{\infty} ; F\right) \cong F$ and the cup product pairing $H^{k}\left(Y_{\infty} ; F\right) \times H^{n-1-k}\left(Y_{\infty}, \partial Y_{\infty} ; F\right) \rightarrow F$ is nonsingular.

In applying this theorem, we will make use of the following two facts, the first essentially due to Milnor and the second to Casson and Gordon. 
LEMMA 4.2 [18]. If $H_{*}(Y) \cong H_{*}\left(S^{1}\right)$ or $H_{*}\left(S^{1} \times S^{n-1}\right)$ then $H_{*}\left(Y_{\infty} ; F\right)$ is finitely generated for all fields $F$.

Proof. Milnor shows this for $H_{*}(Y) \cong H_{*}\left(S^{1}\right)$, but in the second case, $Y=S^{1} \times$ $B^{n-1} \cup_{S^{1} \times S^{n-2}} X$ where now $H_{*}(X)=H_{*}\left(S^{1}\right)$ so the result follows.

LEMMA 4.3 [4, LeMMA 6]. If there is a prime $p$ such that $H_{*}\left(Y_{\infty}, \mathbf{Z}_{p}\right)$ is finite, then $H_{*}\left(Y_{\infty}, \mathbf{Q}\right)$ is finitely generated.

If $H_{*}\left(Y_{\infty} ; F\right)$ and $H_{*}\left(\partial Y_{\infty} ; F\right)$ are finite dimensional then the conclusion of Theorem 4.1 will be summarised by saying $\left(Y_{\infty}, \partial Y_{\infty}\right)$ satisfies duality over $F$. This can be given the usual formulation in terms of cup product with a generator of $H_{n-1}\left(Y_{\infty}, \partial Y_{\infty} ; F\right)$ (an “ $F$-fundamental class"). Using duality we can think of a nonsingular intersection product on homology with the usual properties. So if $Y$ is $(4 k+1)$-dimensional, then $Y_{\infty}$ has a signature $\sigma\left(Y_{\infty}\right)$. Suppose $\phi: H_{1}\left(Y_{\infty}\right) \rightarrow \mathbf{Z}_{p^{r}}$ is a character which induces the cyclic cover $\tilde{Y}_{\infty} \rightarrow Y_{\infty}$.

Proposition 4.4. If $H_{*}\left(Y_{\infty} ; \mathbf{Z}_{p}\right)$ and $H_{*}\left(\partial Y_{\infty} ; \mathbf{Z}_{p}\right)$ are finite, then $\tilde{Y}_{\infty}$ satisfies duality over $\mathbf{Q}$ (and hence over $\mathbf{C}$ ).

Proof. Casson and Gordon [4, Lemma 4] show that $\tilde{Y}_{\infty}$ is a $\mathbf{Z}$-cover of a finite complex; since a cover is a local homeomorphism that complex must be a compact manifold with boundary. Lemma 5 of [4] says that $H_{*}\left(\tilde{Y}_{\infty} ; \mathbf{Z}_{p}\right)$ and $H_{*}\left(\partial \tilde{Y}_{\infty} ; \mathbf{Z}_{p}\right)$ are finite, so by Lemma $4.3, H_{*}\left(\tilde{Y}_{\infty} ; \mathbf{Q}\right)$ and $H_{*}\left(\partial \tilde{Y}_{\infty} ; \mathbf{Q}\right)$ are finite dimensional. Hence $\left(\tilde{Y}_{\infty}, \partial \tilde{Y}_{\infty}\right)$ satisfies duality over $\mathbf{Q}$.

Suppose now $n=2 k+1$, that $H_{*}\left(Y_{\infty} ; \mathbf{Z}_{p}\right)$ and $H_{*}\left(\partial Y_{\infty} ; \mathbf{Z}_{p}\right)$ are finite, and that we have a character $\phi: H_{1}\left(Y_{\infty}\right) \rightarrow \mathbf{Z}_{p^{r}}$. Proposition 4.4 implies that $H_{k}\left(\tilde{Y}_{\infty} ; \mathrm{C}\right)$ is finite dimensional and supports a Hermitian form made from the intersection form just as in the compact case. $H_{k}\left(\tilde{Y}_{\infty} ; \mathbf{C}\right)$ splits into eigenspaces of the action of $\mathbf{Z}_{p^{r}}$ and so we get signatures $\bar{\sigma}\left(Y_{\infty}\right)$. These can be used to define Casson-Gordon invariants $\sigma\left(\partial Y_{\infty}, \phi\right)$ - see below. The signatures $\bar{\sigma}\left(Y_{\infty}\right)$ are connected to the corresponding invariants of compact manifolds. To explain this we need the concept of a section.

Proposition 4.5. Suppose $Y_{\infty} \stackrel{\pi}{\rightarrow} Y^{n}$ is a $\mathbf{Z}$-cover of the compact manifold $Y$ with $Y_{\infty}$ and $\partial Y_{\infty}$ connected. Then there is a compact connected submanifold $\left(N^{n-1}, \partial N\right) \subset$ $\left(Y_{\infty}, \partial Y_{\infty}\right)$ such that

(1) $N$ separates $Y_{\infty}$,

(2) for all fields $F$ over which $\left(Y_{\infty}, \partial Y_{\infty}\right)$ satisfies duality, $[N, \partial N]$ represents an $F$-fundamental class in $H_{n-1}\left(Y_{\infty}, \partial Y_{\infty} ; F\right)$.

Proof. $\pi: Y_{\infty} \rightarrow Y$ is classified by a map $f: Y \rightarrow S^{1}$. After a small homotopy, $1 \in S^{1}$ will be a regular value for $f$ and for $f_{\mid \partial Y}$. Let $M=f^{-1}(1)$. Since $Y_{\infty}$ and $\partial Y_{\infty}$ are connected, the classes $[f] \in H^{1}(Y)\left(=\left[Y, S^{1}\right]\right)$ and $i^{*}[f] \in H^{1}(\partial Y)$ are primitive and so there is a circle $C=\partial Y$ such that $C \cdot \partial M=i(C) \cdot(M)=1$. Tubing together the components of $\partial M$, and then of $M$, gives a connected submanifold $(N, \partial N)$ which hits $C$ (and $i(C)$ ) exactly in one point and which is hence dual to $f$. It follows that $Y_{\infty}$ can be built by cutting $(Y, \partial Y)$ along $(N, \partial N)$ and gluing copies of 
$Y-N$ end to end. Thus (1) is proved. To get (2) note that the lift $(N, \partial N) \subset$ $\left(Y_{\infty}, \partial Y_{\infty}\right)$ intersects a line (i.e. $\left.\pi^{-1}(C)\right)$ in one point and hence is nontrivial in homology. Since $H_{n-1}\left(Y_{\infty}, \partial Y_{\infty} ; F\right) \cong F$, which is a field, [N, $\left.\partial N\right]$ is a generator.

Definition. A submanifold $N \subset Y_{\infty}$ satisfying Proposition 4.5 will be called a section of $Y_{\infty}$.

Note that a relative version of the argument of Proposition 4.5 shows that if we have picked a section $M$ of $\partial Y_{\infty}$ there is a section $N$ of $Y_{\infty}$ extending $M$, that is, $N=\partial M$. The utility of a section is that it enables one to calculate $\sigma\left(Y_{\infty}\right)$ and $\bar{\sigma}\left(Y_{\infty}, \phi\right)$ when the latter is defined.

Lemma 4.6. (a) If $N^{n-1}$ is a section of $Y_{\infty}$ and $Y_{\infty}$ satisfies duality over the field $F$, then $i_{*}: H_{j}(N ; F) \rightarrow H_{j}\left(Y_{\infty} ; F\right)$ is onto.

(b) If $Y_{\infty}$ satisfies duality over $\mathbf{Z}_{p}$ then $i_{*}: H_{1}\left(N ; \mathbf{Z}_{p^{r}}\right) \rightarrow H_{1}\left(Y_{\infty} ; \mathbf{Z}_{p^{r}}\right)$ is onto.

Proof. The first part is the familiar statement used in surgery theory that a degree one map is onto in homology. More formally, let $\mu=i_{*}\left(\mu_{N}\right)$ be an $F$-fundamental class. Given an element $b \in H_{j}\left(Y_{\infty} ; F\right)$ there is a $z \in H^{n-1-j}\left(Y_{\infty} ; \partial Y_{\infty} ; F\right)$ with $z \cap \mu=b$. Then $b=z \cap \mu=z \cap i_{*} \mu_{N}=i_{*}\left(i^{*} z \cap \mu_{N}\right)$ so $i_{*}$ is onto. For $j=1$, the long exact sequence of $\left(Y_{\infty}, N\right)$ says that $H_{1}\left(Y_{\infty}, N ; F\right)=0$. If now $F=\mathbf{Z}_{p}$ (as in part (b)) induction on $r$ using the Bockstein exact sequence

$$
\rightarrow H_{1}\left(Y_{\infty}, \mathbf{Z}_{p}\right) \rightarrow H_{1}\left(Y_{\infty}, N ; \mathbf{Z}_{p^{r}}\right) \rightarrow H_{1}\left(Y_{\infty}, N ; \mathbf{Z}_{p^{r-1}}\right) \rightarrow
$$

shows that $H_{1}\left(Y_{\infty}, N ; \mathbf{Z}_{p^{r}}\right)=0 \forall r$. Hence (b) is proved.

So a character $\phi: H_{1}\left(Y_{\infty}\right) \rightarrow \mathbf{Z}_{p^{r}}$ yields a character $\phi \circ i$ on $H_{1}(N)$; the next theorem relates the signatures associated to $(N, \phi \circ i)$ and $\left(Y_{\infty}, \phi\right)$ and is the central tool of this chapter.

THEOREM 4.7. (a) Suppose $Y_{\infty} \rightarrow Y^{2 k+1}$ is a connected $\mathbf{Z}$-cover with $H_{*}\left(Y_{\infty} ; \mathbf{Q}\right)$ and $H_{*}\left(\partial Y_{\infty} ; \mathbf{Q}\right)$ finitely generated. Then for any section $N$ of $Y_{\infty}, \sigma\left(Y_{\infty}\right)=\sigma(N)$.

(b) Suppose further that $H_{*}\left(Y_{\infty} ; \mathbf{Z}_{p}\right)$ and $H_{*}\left(\partial Y_{\infty} ; \mathbf{Z}_{p}\right)$ are finite. Then for all characters $\phi: H_{1} Y_{\infty} \rightarrow \mathbf{Z}_{p^{r}}, \bar{\sigma}\left(Y_{\infty}, \phi\right)=\bar{\sigma}(N, \phi \circ i)$.

Proof. For all of part (a), Q-coefficients are assumed; part (a) is obvious for $k$ odd (both sides are zero) so assume $k$ is even. By the previous lemma, $H_{*}(N) \rightarrow$ $H_{*}\left(Y_{\infty}\right)$ is onto. A standard argument of surgery theory [3] says that $\sigma\left(Y_{\infty}\right)-\sigma(N)$ $=\sigma(K)$, where $\sigma(K)=$ signature of $\cdot \operatorname{on} \operatorname{ker}\left(i_{*}: H_{k}(N) \rightarrow H_{k}\left(Y_{\infty}\right)\right)$. Since $N$ is a section, it separates $Y_{\infty}$ into two components, $A$ and $B$. The various inclusion maps are summarised in the following diagram.

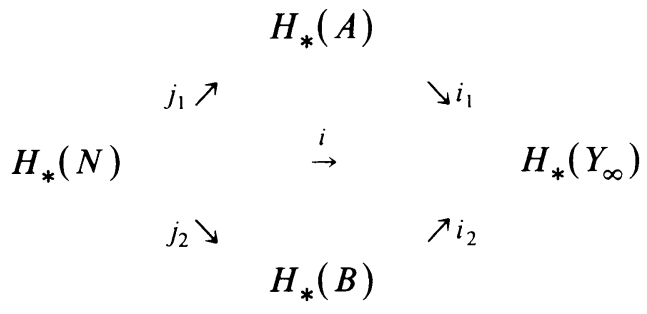


Claim. (i) ker $i=\operatorname{ker} j_{1}+\operatorname{ker} j_{2}(+=$ internal direct sum).

(ii) The intersection form vanishes on $\operatorname{ker} j_{1}$ and $\operatorname{ker} j_{2}$. This claim immediately implies that $\sigma(K)=0$ and hence that $\sigma\left(Y_{\infty}\right)=\sigma(N)$.

Proof of Claim. First consider the relative Mayer-Vietoris sequence of $\left(Y_{\infty}, N\right)$ $=(A, N) \cup(B, N)$. This is

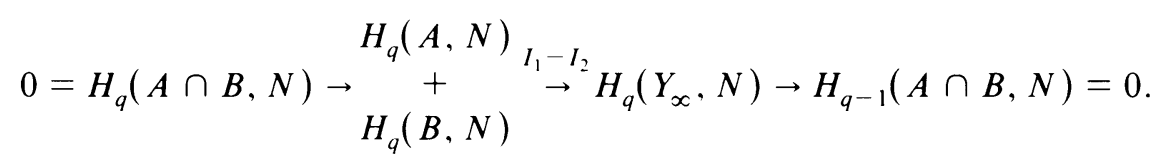

So the inclusions $I_{1}$ and $I_{2}$ are injections. Now compare the long exact sequences of $(A, N)$ and $\left(Y_{\infty}, N\right)$ :

$$
\begin{array}{ccccccc}
H_{q+1}(A, N) & \stackrel{\text { ’ }}{\rightarrow} & H_{q} N & \underset{j_{1}}{\rightarrow} & H_{q} A & \rightarrow & H_{q}(A, N) \\
\downarrow & & \| & & \downarrow i_{1} & & \downarrow I_{1} \\
H_{q+1}\left(Y_{\infty}, N\right) & \stackrel{\text { ว }}{\rightarrow} & H_{q} N & \stackrel{i}{\rightarrow} & H_{q}\left(Y_{\infty}\right) & \stackrel{I}{\rightarrow} & H_{q}\left(Y_{\infty}, N\right)
\end{array}
$$

In the bottom line, $i$ is onto, so $I$ is the zero map. Since $I_{1}$ is injective, a diagram chase shows that $j_{1}$ is onto. The same argument applies to $B$, and so all the sequences split, and we obtain that $\partial: H_{k+1}\left(Y_{\infty}, N\right) \rightarrow \operatorname{ker} i, \partial_{1}: H_{k+1}(A, N) \rightarrow \operatorname{ker} j_{1}$, and $\partial_{2}$ : $H_{k+1}(B, N) \rightarrow \operatorname{ker} j_{2}$ are all isomorphisms. Statement (i) now follows from the above Mayer-Vietoris sequence for $q=k$. Statement (ii) is a standard fact: if $i_{1} c=i_{1} d=0$ then $c=\partial C$ and $d=\partial D$ where $C$ and $D$ are $(k+1)$-chains in general position. $C \cap D=\operatorname{arcs} \cup$ circles; the arcs join algebraically cancelling pairs of points in $c \cap d$. Therefore, $c \cdot d=0$ and the claim is established.

The proof of (b) is basically the same. Since $H_{*}\left(Y_{\infty} ; \mathbf{Z}_{p}\right)$ and $H_{*}\left(\partial Y_{\infty} ; \mathbf{Z}_{p}\right)$ are finite, $H_{*}\left(\tilde{Y}_{\infty} ; \mathrm{C}\right)$ is finite dimensional so $\bar{\sigma}\left(Y_{\infty}, \phi\right)$ is therefore defined. Because of Lemma $4.6(\mathrm{~b}), \tilde{N}$ is connected and is, in fact, a section of $\tilde{Y}_{\infty}$. All the exact sequences in the proof of (a) split into exact sequences of eigenspaces and so the same proof shows that $\bar{\sigma}\left(Y_{\infty}, \phi\right)=\bar{\sigma}(N, \phi \circ i)$.

Part (a) is essentially well known and dates back to Novikov's work on the rational Pontrjagin classes [19]. I am indebted to Larry Taylor for suggesting the relevance of such a proposition to this work.

Now let $Y_{\infty} \rightarrow Y^{2 k}$ be a $\mathbf{Z}$-cover of the closed manifold $Y$ where $H_{*}\left(Y_{\infty} ; \mathbf{Z}_{p}\right)$ is finite and let $\phi: H_{1} Y_{\infty} \rightarrow \mathrm{Z}_{p^{r}}$ be a character. Suppose further that for some $n$ there is a compact manifold $A$ and a $Z$-covering $A_{\infty} \rightarrow A$ with the following properties:

(1) $\partial\left(A_{\infty} \rightarrow A\right)=n \cdot\left(Y_{\infty} \rightarrow Y\right)$.

(2) There is a character $\psi$ on $A_{\infty}$ such that $\partial\left(A_{\infty}, \psi\right) n \cdot\left(Y_{\infty}, \phi\right)$.

(3) $H_{*}\left(A_{\infty} ; \mathbf{Z}_{p}\right)$ is finite.

Definition. Under these hypotheses the Casson-Gordon invariant of $\left(Y_{\infty}, \phi\right)$ is $\sigma\left(Y_{\infty}, \phi\right)=\left(\bar{\sigma}\left(A_{\infty}, \phi\right)-\left(A_{\infty}\right)\right) / n$.

Of course, defining such numbers and calling them invariants does not automatically make them invariants. 
THEOREM 4.8. If there is one manifold $A$ satisfying (1)-(3) then the number $\sigma\left(Y_{\infty}, \phi\right)$, defined using $A$, depends only on $Y$ and the character $\phi$.

Proof. By additivity of the signature and eigenspace signatures, the theorem will follow if we can show that $\bar{\sigma}\left(A_{\infty}, \phi\right)-\sigma\left(A_{\infty}\right)=0$ whenever $A_{\infty} \rightarrow A^{2 k+1}$ is a $\mathbf{Z}$-cover of a closed manifold $A$. (Here $\phi: H_{1} A_{\infty} \rightarrow \mathbf{Z}_{p^{r}}$ and we assume $H_{*}\left(A_{\infty} ; \mathbf{Z}_{p}\right)$ is finite.) $A$ has a section $N^{2 k}$ and by Theorem 4.7, $\bar{\sigma}\left(A_{\infty}, \phi\right)=\bar{\sigma}(N, \phi \circ i)$ and $\sigma\left(A_{\infty}\right)=\sigma(N)$. But now the bordism argument $[\mathbf{8}, \S 1]$ that the usual Casson-Gordon invariants are well defined says that $\bar{\sigma}(N, \phi \circ i)-\sigma(N)=0$.

For a given $\left(Y_{\infty}, \phi\right)$, if there is an $\left(A_{\infty}, \phi\right)$ satisfying (1)-(3) then we say that $\sigma\left(Y_{\infty}, \phi\right)$ can be defined. It is conceivable that $\sigma\left(Y_{\infty}, \phi\right)$ can always be defined but this is far from obvious. Note that when $\sigma\left(Y_{\infty}, \phi\right)$ can be defined, it is equal to $\sigma(N, \phi \circ i)$ where $N$ is any section of $Y_{\infty}$.

There is a somewhat simpler invariant for knots in $S^{4}$ which is always defined. It is essentially the $\mu$-invariant of a Seifert surface for $K$; however, we must be a little careful about framings.

Definition. Let $\left(S^{4}, K\right)$ be a knot, $\left(B^{5}, D^{3}\right)$ any slice of $K$, and $A_{\infty}$ the Z-cover of $B^{5}-D^{3} \times \dot{D}^{2}$. Then $\mu(K)=\sigma\left(A_{\infty}\right)(\bmod 16)$.

We must show this is well defined.

THEOREM 4.9. $\mu(K)$ does not depend on the choice of slice disc.

Proof. Suppose we had two slices $D$ and $D^{\prime}$. They fit together to give a knot $\left(S^{5}, J\right)$, with Seifert surface $V_{0}^{4}$. The Z-cover of $J$ is $A_{\infty} \cup-A_{\infty}^{\prime}$ so $\sigma\left(A_{\infty}\right)-\sigma\left(A_{\infty}^{\prime}\right)$ $=\sigma\left(V_{0}\right)$ by Theorem 4.7. But $V_{0}$ is a spin-manifold, so by Rochlin's Theorem $16 \mid \sigma\left(V_{0}\right)$. Hence, $\sigma\left(A_{\infty}\right) \equiv \sigma\left(A_{\infty}\right) \bmod 16$.

Any Seifert surface $M_{0}$ lies in the exterior of $K$ and hence in $\mathbf{R}^{4}=S^{4}$-point. Hence, $M_{0}$ has a framing $F_{0}$ which is the restriction of the unique framing on $\mathbf{R}^{4}$. Then one can use the above argument to show that $\mu(K)=\mu\left(M, F_{0}\right)$ for any Seifert surface.

We are now in a position to find some non-doubly slice knots. The following notation will be in use for the rest of this section. $\left(S^{2 k+2}, K\right)$ is a knot with $\mathbf{Z}$-cover $X_{\infty}$; the boundary of $X_{\infty}$ is $S^{2 k} \times \mathbf{R}$ which we identify with $K \times \mathbf{R}$. Let $Y$ be the result of surgery on $K$; then $Y$ has an infinite cyclic cover $Y_{\infty}$ which equals $X_{\infty} \cup B^{2 k+1} \times \mathbf{R}$. The $k n o t K$ is a slice knot [16], and for a slice $D^{2 k+1} \subset B^{2 k+3}$ we set $A=B^{2 k+3}-D^{2 k+1} \times \dot{D}^{2}$ and $A_{\infty}=$ the $\mathbf{Z}$-cover of $A$. Note that $\partial\left(A_{\infty} \rightarrow A\right)=$ $Y_{\infty} \rightarrow Y$ and that by Lemma $4.2, H_{*}\left(Y_{\infty} ; F\right)$ and $H_{*}\left(A_{\infty} ; F\right)$ are finitely generated for any field $F$.

The essence of the technique is contained in the following result which gives a new criterion for double null-concordance for a knot in $S^{4}$. Recall [9] that if $M^{3} \subset S^{4}$, then $M$ has a framing $F$ for which $\mu(M, F)=0$. In the same spirit, we have

THEOREM 4.10. If $K$ is doubly slice, then $\mu(K)=0$. Equivalently, any Seifert surface has a framing $F_{0}$ for which $\mu\left(M, F_{0}\right)=0$. 
Proof. By the previous result, we can use the $\mathbf{Z}$-cover of any disc to calculate $\mu(K)$. So choose $A_{\infty}=\mathbf{Z}$-cover of one disc making up the unknotted $S^{2}$ whose slice is $K$. The Mayer-Vietoris sequence shows that $i_{*}: H_{2}\left(Y_{\infty} ; \mathbf{Q}\right) \rightarrow H_{2}\left(A_{\infty} ; \mathbf{Q}\right)$ is onto. But it is easy to see that the intersection form vanishes identically on image $\left(i_{*}\right)$. Therefore $\mu(K) \equiv \sigma\left(A_{\infty}\right)=0$. The second statement follows from the remark after Theorem 4.9.

COROLlARY 4.11. There are algebraically but not geometrically doubly slice knots in $S^{4}$.

Proof. Let $K$ be 5-twist spin of the trefoil knot. $K$ is a fibered knot with fiber $M_{0}=$ Poincaré homology sphere. Since $M$ has no homology, the linking form is empty (hence hyperbolic) so $K$ is algebraically doubly slice. But $\mu(M) \neq 0$ so $K$ is not doubly slice.

In a similar way we can reformulate the obstructions of Gilmer and Livingston to imbedding $M^{3}$ in $S^{4}$ as obstructions to a knot in $S^{4}$ being doubly slice.

THEOREM 4.12. Let $K$ be a knot in $S^{4}$ and suppose $H_{1}\left(Y_{\infty} ; \mathbf{Q}\right)=0$. If $K$ is doubly slice, then $H_{1}\left(Y_{\infty}\right)=G+G^{\prime}$ (as $\Lambda$-modules) where (1) $G \cong G^{\prime}$, (2) $L_{G G}=L_{G G^{\prime}}=0$ ( $L$ is the Levine form) and (3) for all characters $\phi: H_{1}\left(Y_{\infty}\right) \rightarrow \mathbf{Z}_{p^{r}}$ vanishing on $G$ or $G^{\prime}$, $\sigma\left(Y_{\infty}, \phi\right)$ is defined and satisfies

$$
\left|\sigma\left(Y_{\infty}, \phi\right)\right|+\bar{\beta}_{1}\left(Y_{\infty} ; \phi\right)<\operatorname{dim} H_{1}\left(Y_{\infty} ; \mathbf{Z}_{p}\right) .
$$

Proof. If $K$ is doubly slice, then $Y_{\infty} \subset S^{4} \times \mathbf{R}$, which then separates into $A_{\infty} \cup A_{\infty}^{\prime}$. Note that $A_{\infty}$ and $A_{\infty}^{\prime}$ are both $\mathbf{Z}$-covers of homology circles, so that $H_{1}\left(A_{\infty} ; \mathbf{Z}_{p^{n}}\right)$ is finite for all $n$ as is $H_{1}\left(A_{\infty}^{\prime} ; \mathbf{Z}_{p^{n}}\right)$. If we let

$$
G=\operatorname{ker}\left[H_{1}\left(X_{\infty}\right) \rightarrow H_{1}\left(A_{\infty}\right)\right]
$$

and $G^{\prime}=$ the other kernel, then the Mayer-Vietoris sequence shows that $H_{1}\left(X_{\infty}\right)=$ $G+G^{\prime}$ over $\Lambda$. Stoltzfus' argument $[22,23]$ that $K$ is algebraically doubly slice says exactly that (1) and (2) must hold.

Suppose $\phi_{G G}=0$, then $\phi$ extends over $A_{\infty}$, and so $\sigma\left(X_{\infty}, \phi\right)$ is defined and equals $\bar{\sigma}\left(A_{\infty}, \phi\right)-\sigma\left(A_{\infty}\right)$. Gilmer and Livingston [9], in a similar situation with $Y_{\infty}$ replaced by an honest closed 3-manifold and $A_{\infty}$ replaced by a compact 4-manifold, used duality over $\mathbf{C}$ and a Smith theory argument to conclude that $(*)$ holds. But duality holds, and we noted in the introduction that the relevant results about finite cyclic covers (Propositions 1.4-1.6) are valid in the current context.

In practice it is generally easier to calculate the Casson-Gordon invariants of a section than to calculate $\sigma\left(Y_{\infty}, \phi\right)$. So Theorem 4.12 can be viewed as a bound on $\sigma(M, \phi \circ i)$ where $M_{0}$ is any Seifert surface for $K$. If $K$ is a fibered knot with fiber $M_{0}$, then $Y_{\infty} \cong M \times \mathbf{R}$, and Theorem 4.12 specialises to

Corollary 4.13. Suppose $K \subset S^{4}$ is a fibered knot with fiber $M_{0}$. If $K$ is doubly slice, then the conclusion of Theorem 4.12 holds with $Y_{\infty}$ replaced by $M$. 
Corollary 4.13, which presents an obstruction to imbedding $M \times \mathbf{R}$ in $S^{4} \times \mathbf{R}$, is formally the same as the obstruction to embedding $M$ in $S^{4}$ found by Livingston and Gilmer.

It is well known [29] that 2-twist spinning the rational knot $q / m$ ( $m$ odd) gives a fibered knot in $S^{4}$, denoted $\tau(m ; q)$, whose fiber is a lens space $L(m ; q, 1)$.

Corollary 4.14. Let $\tau(m ; q)$ and $\tau\left(m ; q^{\prime}\right)$ be twist-spun rational knots. Then

(a) $\tau \#-\tau^{\prime}$ is algebraically double slice iff for some $c, q^{ \pm 1} q^{\prime} \equiv c^{2} \bmod m$ (i.e. the fibers are homotopy equivalent preserving orientation).

(b) If $m$ is a prime power, then $\tau$ - $\tau^{\prime}$ is doubly slice if and only if $\tau=\tau^{\prime}$.

Proof. (a) This follows from the calculation of the linking form of a lens space: $(q / m)+\left(-q^{\prime} / m\right)$ is hyperbolic if and only if $q^{ \pm 1} q^{\prime}$ is a square mod $m$. Since the action of $t$ on $H_{1}\left(L \#-L^{\prime}\right)$ is multiplication by -1 , any splitting of the linking form of the fiber is $t$-invariant and so gives rise to a splitting of the Levine form.

(b) Gilmer and Livingston show that for $m=$ prime power, (1)-(3) of Theorem 4.12 imply that the fibers $L$ and $L^{\prime}$ are diffeomorphic preserving orientation. Hence the original rational knots are the same, so $\tau=\tau^{\prime}$.

At this point it should be fairly apparent how to find obstructions to double null concordance in all even dimensions: we should translate the nonimbedding Theorem 2.2 .

THEOREM 4.15. Let $K$ be a knot in $S^{2 k+2}$ with $H_{1}\left(Y_{\infty} ; \mathbf{Q}\right)=H_{k}\left(Y_{\infty} ; \mathbf{Q}\right)=0$. If $K$ is doubly slice, then $H_{*}\left(Y_{\infty}\right)=G_{*}+G_{*}^{\prime}($ over $\Lambda)$ with (1) $G_{k} \cong G_{k}^{\prime},(2) L_{G}=L_{\mid G^{\prime}}=0$, and (3) for all characters $\phi: H_{1}\left(Y_{\infty}\right) \rightarrow \mathbf{Z}_{p^{r}}$ with $\phi_{*}$ vanishing on $G_{*}$ or $G_{*}^{\prime}, \sigma\left(Y_{\infty}, \phi\right)$ is defined and satisfies $\left|\sigma\left(Y_{\infty}, \phi\right)\right| \leqslant \operatorname{dim} H_{k+1}\left(Y_{\infty}, \mathbf{Z}_{p}\right)$.

Proof. The proof is the same as that of Theorem 2.2. If $K$ is doubly slice, then $Y_{\infty} \subset S^{2 k+2} \times \mathbf{R}$, and we can use one component of $S^{2 k+2} \times \mathbf{R}-Y_{\infty}$ to define and evaluate $\sigma\left(Y_{\infty}, \phi\right)$. But by the Mayer-Vietoris sequence and Smith theory (Proposition 1.5), $\sigma\left(Y_{\infty}, \phi\right)$ is bounded by $\operatorname{dim} H_{k+1}\left(Y_{\infty}, \mathbf{Z}_{p}\right)$.

Again, one can interpret Theorem 4.15 as giving a bound on $\sigma(M, \phi)$ for $M_{0}$ any Seifert surface of $K$. Recall the knots $K(J, q) \subset S^{2 k+1}$ constructed in $\S 3$. Taking the 2-twist spin of $K(J, q)$ gives a knot $L(J, q) \subset S^{2 k+2}$.

COROllaRy 4.16. If $L$ is doubly slice and $2 q+1=$ prime power then

$$
\left\{\begin{array}{l}
\sigma_{r / 2 q+1}(J)=0 \quad(k \text { odd }), \\
\sigma_{r / 2 q+1}(J)+2^{2 k-2} \frac{2 q+1-2 r}{2 q+1} \sigma_{1 / 2}(J)=0 \quad \text { (k even). }
\end{array}\right.
$$

Proof. The fiber $M$ of $L$ is the 2-fold branched cover of $S^{2 k+1}$ branched along $K$. The above expressions give the Casson-Gordon invariants of $M$ and hence of $Y_{\infty}$. But $Y_{\infty}$ has no middle-dimensional homology, so these must vanish.

Note that $L(J, q)$ is algebraically doubly slice. So with correct choice of $J$, Corollary 4.16 combines with Corollary 3.3 and the examples of Gilmer and Livingston to yield 
THEOREM 4.17. In every dimension, there are slice knots which algebraically but not geometrically doubly slice.

It should be pointed out that these results do not settle a related issue. This arises when one uses double null-concordance to define a group $\mathrm{CH}_{n}$. The obstructions defined by Sumners and Stoltzfus provide homomorphisms onto algebraically defined groups $\mathrm{CH}^{\varepsilon}(\mathbf{Z})$ and $\mathrm{CH}^{\varepsilon}(\mathbf{Q} / \mathbf{Z})$ in odd and even dimensions, respectively $(\varepsilon= \pm 1)$. One might suppose that Theorem 4.17 says that the kernels of these maps are nonzero, or even infinitely generated. However, this is not so. The problem is in how $\mathrm{CH}_{n}$ is defined (cf. [23]): $\left(S^{n+2}, K\right)=0$ in $\mathrm{CH}_{n}$ if there is a doubly slice knot $K^{\prime}$ such that $K \# K^{\prime}$ is doubly slice, or in other words if $K$ is stably doubly slice.

It is conceivable that a knot which is shown not to be doubly slice by our methods might actually be stably doubly slice. In view of the general viewpoint of this paper the following question seems the right place to start.

Question. If $N^{n}$ imbeds in $S^{n+1}$, and $N \# M \subset S^{n+1}$, does $M$ imbed in $S^{n+1}$ ?

REMARK. T. Cochran and J. Levine have recently pointed out that the $\mu$-invariant must vanish for stably doubly slice knots in $S^{4}$. Hence the kernel of Stoltzfus' map $\mathrm{CH}_{2} \rightarrow \mathrm{CH}^{+1}(\mathbf{Q} / \mathbf{Z})$ is nontrivial.

5. Further applications. In this section we give some further applications of the Casson-Gordon invariants to high-dimensional knot theory. The first is to the study of ribbon knots and the second is to amphicheirality and invertibility of knots.

Definition. A ribbon in $S^{n+2}$ is an immersion $f: D^{n+1} \rightarrow S^{n+2}$ with only transverse double points, and such that $S(f)=\left\{D_{i}^{n}\right\} \cup\left\{\bar{D}_{i}^{n}\right\}$ where $D_{i} \cap \bar{D}_{j}=\varnothing$ $\forall i, j$, and $D_{i}, \bar{D}_{j}$ have disjoint images if $i \neq j$. Furthermore, $f\left(D_{i}\right)=f\left(\overline{D_{i}}\right)$ and $D_{i} \subset$ int $D^{n+1}$ while the $\bar{D}_{i}^{n}$ are properly imbedded.

The boundary of a ribbon is imbedded and will be called a ribbon knot. The fundamental geometric fact about ribbon knots is the following proposition, which is quite well known.

Proposition 5.1. Let $\left(S^{n+2}, K\right)$ be a ribbon knot. Then $K$ is a slice knot. In fact, $K$ bounds an (imbedded) disc $D^{n+1}$ in $B^{n+3}$ such that $B^{n+3}-D^{n+1} \times \dot{D}^{2}$ has a handle decomposition with only $0-, 1$ - and 2-handles.

Proof. $f\left(D_{i}\right)$ lies on two intersecting sheets, and it is in the interior of one of them. On that sheet, push a small regular neighborhood of $f\left(D_{i}\right)$ into $B^{n+3}$. Under the radial Morse function on $B^{n+3}$, the resulting $D^{n+1}$ has only 0 and 1-handles; the dual decomposition of the exterior is then as described.

Another way of stating the conclusion is to say that $B^{n+3}-D^{n+1} \times \dot{D}^{2}$ is built from its boundary ( = surgery on $K)$ by adding $(n+1)$ - and $(n+2)$-handles and an $(n+3)$-handle. The other geometric fact we need about ribbon knots is the following theorem, due to Yanagawa for $n=2$.

THEOREM 5.2. A ribbon knot in $S^{n+2}$ has a Seifert surface diffeomorphic to $\#_{k} S^{n} \times S^{1}-D^{n+1}$. 
Proof. We show how to replace each singularity $f\left(D^{n}\right)$ of a ribbon by an imbedded 1-handle. The construction is completely local and is summarised by the following pictures of a standard model (Figures (a)-(f)):

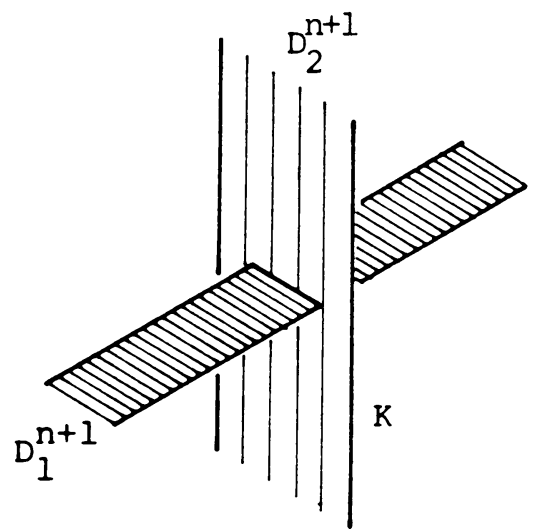

(a)

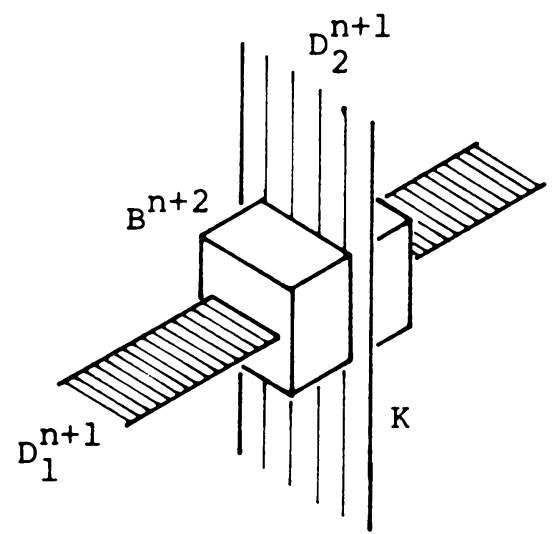

(b)

Remove the ball $B^{n+2}$ pictured in (b). The local pictures on the two sheets of $D^{n+2}$ now look like:

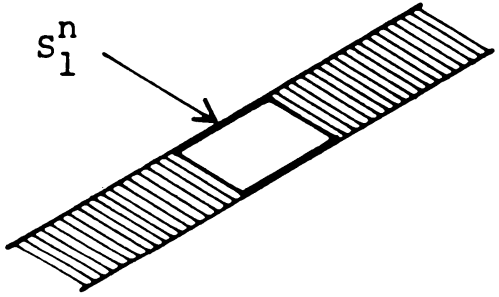

(c)

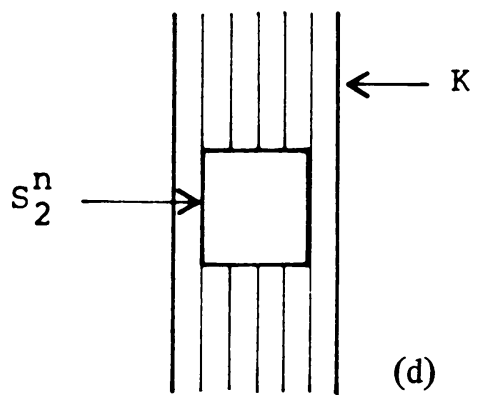

(d)

The boundaries of the holes left by removing $B^{n+2} \cap f$ (int $D^{n+1}$ ) in the sheets (Figures (c) and (d)) are both diffeomorphic to $S^{n}$; if we see how to add an annulus $S^{n} \times I$ to the picture we will have removed the singularity $f\left(D^{n}\right)$ at the expense of connect-summing with $S^{n} \times S^{1}$. But the spheres are unlinked inside $B^{n+2}: S_{1}^{n}$ bounds a disc in the interior while $S_{2}^{n}$ sits on the boundary and bounds a disc. Hence we can isotop Figure (e) to look like Figure (f) where it is obvious that one can add the annulus $S^{n} \times I$.

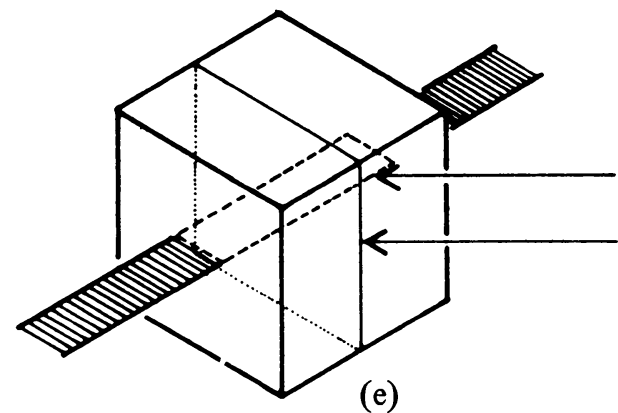

(e)

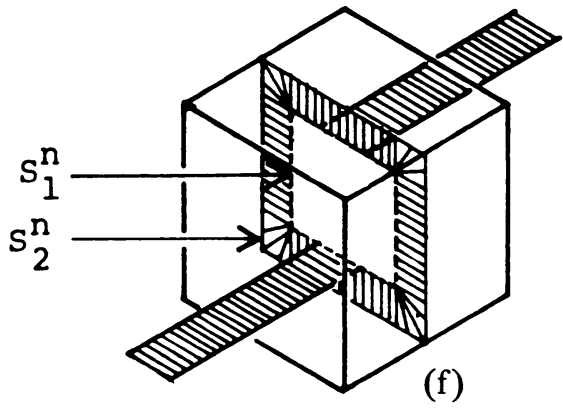

(f) 
This geometric picture gives a strong obstruction to a knot being ribbon in terms of the invariants defined in $\S 4$.

THEOREM 5.3. Suppose $K \subset S^{2 q+2}$ is a ribbon knot. Then

(a) For all characters $\phi: H_{1}\left(Y_{\infty}\right) \rightarrow \mathbf{Z}_{p^{r}}, \sigma\left(Y_{\infty}, \phi\right)$ is defined and is zero.

(b) For $q=1, \mu(K)=0$.

Proof. (a) Let $A=$ the exterior of the slice for $K$ built in Proposition 5.1. Since $A=Y \cup$ handles of index $\geqslant 2 q+1, A_{\infty}=Y_{\infty} \cup$ handles of index $\geqslant 2 q+1$. It follows that $H_{1}\left(Y_{\infty}\right) \cong H_{1}\left(A_{\infty}\right)$ so that any character on $Y_{\infty}$ extends over $A_{\infty}$ which can be used to define $\sigma\left(Y_{\infty}, \phi\right)$. In $\S 4$ it was observed that whenever $\sigma\left(Y_{\infty}, \phi\right)$ is defined, it equals $\sigma(N, \phi \circ i)$ for $N$ any section of $Y_{\infty}$, e.g. a Seifert surface for the knot. By 5.2 we have that $\sigma\left(Y_{\infty}, \phi\right)=\sigma\left(\#_{k} S^{2 q} \times S^{1}, \phi \circ i\right)$. But $\sigma\left(\#_{k} S^{n} \times S^{1}, \psi\right)$ $=0$ for any $\psi$, because $\left(\#_{k} S^{n} \times S^{1}, \phi\right)=\partial\left(\natural_{k} D^{n+1} \times S^{1}, \psi\right)$ and $\natural_{k} D^{n+1} \times S^{1}$ has no signatures.

(b) The same argument applies: $\mu(K)=\mu\left(\#_{k} S^{2} \times S^{1}, F_{0}\right)$ for $F_{0}$ some framing. But $\#_{k} S^{2} \times S^{1}=\partial\left(\natural_{k} D^{3} \times S^{1}\right)$ and any framing extends over $\natural_{k} D^{3} \times S^{1}$. Therefore $\mu(K)=0$.

EXAMPLES. We have constructed many examples of knots with nontrivial CassonGordon invariants; it follows that none of them are ribbon. For instance, none of the 2-twist spun rational knots are ribbon because some Casson-Gordon invariant of a given lens space is nonzero. These examples are all detected by the method of Hitt [12] who observes that $H_{1}(Y)$ must be torsion free if $K$ is a ribbon knot. Examples in $S^{4}$ not detected by Hitt's method arise from the $\mu$-invariant as in 5.3(b) above. For example, the 5-twist spin of the trefoil, whose fiber is the Poincare homology sphere, cannot be ribbon.

As a final application, we extend the results of Farber [6] and Hillman [11] on invertibility and amphicheirality of even-dimensional knots. Both of these authors use the Farber-Levine linking form to detect such symmetries; we use the CassonGordon invariants. All knots in this section are considered as oriented knots, and there is a fixed orientation on the ambient sphere. Recall that oriented knots are equivalent if they are isotopic, or equivalently if there is an orientation preserving diffeomorphism of $S^{n+2}$ taking one onto the other so that the orientations agree.

Definition. $r K=$ image of $K$ (and its orientation) under a reflection in $S^{n+2}$. $K \rho=K$ with its orientation reversed, and $-K=r K \rho$ is the usual concordance inverse. $K$ is invertible if it is equivalent to $K \rho$, and + (or - ) amphicheiral if it is equivalent to $r K$ (or $-K$ ).

Hillman [11] shows that the Levine form is an obstruction to amphicheirality and invertibility, and that in the odd simple case it is the complete obstruction. In the nonsimple case, as one might expect, it is no longer the sole obstruction. Our results are stated for fibered knots only, but they extend to cover the nonfibered case as well. If $K$ is a fibered knot and $\phi: M \rightarrow M$, the action of the monodromy on the capped-off fiber, then $\phi_{*}=t$ defines a $\Lambda=\mathbf{Z}\left[t, t^{-1}\right]$ module structure on $H_{*}(M)$. As a $\Lambda$-module, $H_{*}(M)$ can be identified with $H_{*}\left(Y_{\infty}\right)$ since $Y_{\infty} \cong M \times \mathbf{R}$. 
THEOREM 5.4. Let $K$ be an even-dimensional fibered knot with fiber $M_{0}$ and monodromy $t$.

(a) If $K$ is invertible, then there is a $\Lambda$-antiautomorphism $\theta\left(\theta(t x)=t^{-1} \theta(x)\right)$ of $H_{*}(M)$ such that (i) $\lambda(\theta x, \theta y)=-\lambda(x, y)$ and (ii) $\forall \chi: H_{1}(M) \rightarrow \mathbf{Z}_{d}, \sigma(M, \chi)=$ $-\sigma(M, \chi \circ \theta)$.

(b) If $K$ is (+) amphicheiral, then there is a $\Lambda$-antiautomorphism $\theta$ of $H_{*}(M)$ such that (i) $\lambda(\theta x, \theta y)=\lambda(x, y)$ and (ii) $\forall \chi: H_{1}(M) \rightarrow \mathbf{Z}_{d}, \sigma(M, \chi)=\sigma(M, \chi \circ \theta)$.

(c) If $K$ is (-) amphicheiral, then there is a $\Lambda$-automorphism $\theta$ such that (i) $\lambda(\theta x, \theta y)=-\lambda(x, y)$ and (ii) $\forall \chi: H_{1}(M) \rightarrow \mathbf{Z}_{d}, \sigma(M, \chi)=-\sigma(M, \chi \circ \theta)$.

Remark. Parts (i) of (a)-(c) are the obstructions observed by Hillman [11].

PROOF. We prove the criterion for invertibility; the other two are similar. Consider an isotopy of $K$ onto $K \rho$ and let $f\left(S^{2 q+2}, K\right) \rightarrow\left(S^{2 q+2}, K \rho\right)$ be the diffeomorphism at the end of the isotopy; $f$ lifts to a diffeomorphism $F$ of $Y_{\infty}=M \times \mathbf{R}$. Now $f$ is orientation preserving, so it reverses the meridian of $K$, hence $\theta=F_{*}$ reverses the action of $T$. In other words, $\theta$ is an antiautomorphism of $H_{*}(M)$.

Look now at $F(M) \subset M \times \mathbf{R}$; we can assume it is disjoint from a copy of $M \subset M \times \mathbf{R}$. Then $M$ and $F(M)$ cobound a manifold $V$ which is an $h$-cobordism. The map induced on the homology of $M$ by retracting $V$ onto $M$ is exactly $F_{*}$. Since $f$ preserved the global orientation but reversed the meridian it follows that the restriction of the retraction to the boundary reverses the orientation of $M$. Part (i) follows immediately since switching the orientation of $M$ switches the linking form. Part (ii) also follows since if $W$ is a manifold which one uses to calculate $\sigma(M, \psi)$, (i.e. $\partial(W, \phi)=n \cdot(M, \chi))$ then $W \cup n \cdot V$ will calculate that $\sigma\left(-M, \chi \circ F_{*}\right)=$ $\sigma(M, \chi)$. But $\sigma\left(-M, \chi \circ F_{*}\right)=-\sigma\left(M, \chi \circ F_{*}\right)$.

Parts (b) and (c) are proved along the same lines; one needs only to keep track of whether $F$ preserves or reverses the action of $t$ and preserves or reverses the orientation of $M$.

Many of the knots investigated in the previous sections can be shown to be noninvertible or non-(-)amphicheiral using Theorem 5.4.

EXAMPLE. Let $L \subset S^{2 k+2}$ be the 2-twist spin of the knot $K(J, q)$ constructed in §3. $L$ is fibered with fiber $M=$ the 2 -fold branched cover of $K(J, q)$, and the monodromy is the canonical covering translation $t: M \rightarrow M$. Recall that $H_{1}(M)=$ $\mathbf{Z}_{2 q+1}$ with generator $M=$ a meridian coming from the surgery picture as described in $\S 3$. The following lemma calculates $t_{*}$ on $H_{*} M$; it is elementary but does not seem to be widely known.

LEMMA 5.5. If $N \rightarrow S^{n+2}$ is the 2-fold branched cover of a knot $K$ and $t: N \rightarrow N$ the canonical covering translation, then $t_{*}: H_{j}(N) \rightarrow H_{j}(N)(0<j<n+2)$ is multiplication by -1 .

Proof. By Zeeman's twist-spinning theorem, $X=S^{1} \times{ }_{t} N_{0}$ is a knot complement and so has the homology of a circle. The Wang sequence reduces to

$$
0 \rightarrow H_{j}(N) \stackrel{t_{*}-I}{\rightarrow} H_{j}(N) \rightarrow 0 \text { for } 1 \leqslant j \leqslant n+1 .
$$


But $t$ has order 2, so $0=t_{*}^{2}-I=\left(t_{*}-I\right)\left(t_{*}+I\right)$. By the above sequence, $t_{*}-I$ is invertible, so $t_{*}=-I$.

So in our case (i.e. $t_{*}=t_{*}^{-1}=-I$ ) there is no distinction between $\Lambda$-automorphism and antiautomorphism. For instance, $\theta=\mathrm{id}$ is a $\Lambda$-antiautomorphism satisfying 5.4(b). So we have no obstruction to $K$ being (+) amphicheiral. However, if $J$ is chosen correctly, then $K$ is neither (-) amphicheiral nor invertible: Let $\chi: H_{1} M \rightarrow$ $\mathbf{Z}_{2 q+1}$ be the character taking $m$ to 1 . It was shown in $\$ 3$ that the Casson-Gordon invariants of $(M, \chi)$ are

$$
\sigma(M, r \chi)=\left\{\begin{array}{l}
\sigma_{r / 2 q+1}(J) \quad(k \text { odd }) \\
\sigma_{r / 2 q+1}(J)+2^{2 k-2} \frac{2 q+1-2}{2 q+1} \sigma_{1 / 2}(J) \quad(k \text { even }) .
\end{array}\right.
$$

If $L$ were invertible or (-) amphicheiral, then for some automorphism $\theta$ of $H_{*} M$, $\sigma(M, \chi)=-\sigma(M, \chi \circ \theta)=-\sigma(M, r \chi)$ for some $r$. Hence, if $J$ is chosen so that $\sigma_{1 / 2}(J) \geqslant 0$ and $\sigma_{r / 2 q+1}(J)>0$ for all $r$, the resulting $L$ cannot be invertible or $(-)$ amphicheiral.

There are also examples in dimension four.

ExAmple. Let $K$ be the 2-twist spin of the rational knot $\frac{1}{5} . K$ is fibered with fiber $L_{0}(5,1)$ and $t: L(5,1) \rightarrow L(5,1)$ induces multiplication by -1 on $H_{1}(L(5,1))$. The linking form is given by $\lambda(g, g)=\frac{1}{5}$, so there are indeed automorphisms $\theta$ such that $\lambda(\theta g, \theta g)=-\lambda(g, g)$ : they are given by $\theta_{1}(g)=2 g, \theta_{2}(g)=3 g$. Since $t_{*}=-1, \theta_{1}$ and $\theta_{2}$ are antiautomorphisms as well. If $\phi: H_{1}(L) \rightarrow \mathbf{Z}_{5}$ takes $g$ to 1 , then one calculates easily that $\sigma(L, \phi)=-3 / 5, \sigma\left(L, \phi \circ \theta_{1}\right)=\sigma(L, 2 \phi)=-7 / 5$ and $\sigma\left(L, \phi \circ \theta_{2}\right)=\sigma(L, 3 \phi)-7 / 5$. Hence, $K$ cannot be invertible or $(-)$ amphicheiral.

Once again, there is no obstruction to $K$ being $(+)$ amphicheiral. Other more involved computations with twist-spin knots have revealed many knots which satisfy our criterion for $(+)$ amphicheirality. We close with a conjecture that would explain the results of those computations.

Conjecture. Any twist-spun knot is (+) amphicheiral.

AcKNowledgement. The material in this paper is based on my thesis; I would like to thank my advisor, Rob Kirby, for his help and good advice over the years. I also thank Larry Taylor and Pat Gilmer for many helpful conversations about this work.

\section{BIBLIOGRAPHY}

1. S. Akbulut and R. Kirby, Branched covers of surfaces in 4-manifolds, Math. Ann. 252 (1980), $111-132$.

2. M. F. Atiyah and I. M. Singer, The index of elliptic operators. III, Ann. of Math. (2) 87 (1968), 546-604.

3. W. Browder, Surgery on simply-connected manifolds, Springer-Verlag, New York, 1972.

4. A. Casson and C. Gordon, Cobordism of classical knots, preprint, Orsay, 1975.

5. __ On slice knots in dimension three, Proc. Sympos. Pure Math., vol. 32, Amer. Math. Soc., Providence, R.I., 1978.

6. M. S. Farber, Duality in an infinite cyclic covering and even-dimensional knots, Math. USSR Izv. 11 (1978), 749-782. 
7. R. H. Fox, A quick trip through knot theory, Topology of 3-Manifolds (M. K. Fort, Jr., ed.), Prentice-Hall, Englewood Cliffs, N.J., 1963.

8. P. Gilmer, Configurations of surfaces in 4-manifolds, Trans. Amer. Math. Soc. 264 (1981), 353-380.

9. P. Gilmer and C. Livingston, On embedding 3-manifolds in 4-space, preprint, 1981.

10. W. Hantzsche, Einlagerung von Mannigfeltigkeiten in Euklidische Raume, Math. Z. 43 (1938), 38-58.

11. J. Hillman, Finite knot modules and the factorization of certain simple knots, Math. Ann. 257 (1981), 261-274.

12. L. Hitt, Examples of higher-dimensional slice knots which are not ribbon knots, Proc. Amer. Math. Soc. 77 (1979), 291-297.

13. L. Kauffman, Open books, branched covers, and knot periodicity, Topology 13 (1974), 143-166.

14. C. Kearton, Simple knots which are doubly null-concordant, Proc. Amer. Math. Soc. 52 (1975), 471-472.

15. __ An algebraic classification of some even-dimensional knots, Topology 15 (1975), 363-373.

16. M. Kervaire, Les noeuds des dimensions superieures, Bull. Math. Soc. France 93 (1965), 225-271.

17. J. Levine, Knot modules. I, Trans. Amer. Math. Soc. 229 (1977), 1-50.

18. J. Milnor, Infinite cyclic coverings, Topology of Manifolds (J. Hocking, ed.), Prindle, Weber \& Schmidt, Boston, Mass., 1968.

19. S. P. Novikov, On the homotopy and topological invariance of some rational Pontrjagin classes, Soviet Math. Dokl. 6 (1965), 854-857.

20. D. Ruberman, Imbedding punctured lens spaces and connected sums, Pacific J. Math. (to appear).

21. E. Spanier, Algebraic topology, McGraw-Hill, New York, 1966.

22. N. Stoltzfus, Isometries of inner product spaces and their geometric applications, Geometric Topology (J. C. Cantrell, ed.), Academic Press, New York, 1979.

23. Algebraic computations of the integral concordance and double null concordance groups of knots, Knot Theory, Lecture Notes in Math., vol. 685, (J. C. Hausmann, ed.), Springer-Verlag, New York, 1978.

24. D. W. Sumners, Invertible knot cobordisms, Topology of Manifolds (J. C. Cantrell and C. H. Edwards, eds.), Markham, Chicago 1970.

25. __ Invertible knot cobordisms, Comment. Math. Helv. 46 (1971), 240-256.

26. E. Thomas and J. Wood, On manifolds representing homology classes in codimension 2, Invent. Math. 25 (1974), 63-89.

27. T. Yanagawa, Ribbon knots. I: the bounding manifold, Osaka J. Math. 6 (1969), 447-464.

28. D. Zagier, Equivariant Pontrjagin classes and applications to orbit spaces, Lecture Notes in Math., vol. 290, Springer-Verlag, New York, 1972.

29. E. C. Zeeman, Twisting spun knots, Trans. Amer. Math. Soc. 115 (1965), 471-495.

Department of Mathematics, New York University, Courant Institute of Mathematical SCIENCES, NEW YORK, NEW YORK 10012 